\title{
Requirement of cytochrome $c$ for apoptosis in human cells
}

\author{
A Matapurkar ${ }^{1,2}$ and Y Lazebnik ${ }^{\star, 1}$ \\ 1 Cold Spring Harbor Laboratory, One Bungtown Rd, Cold Spring Harbor, NY \\ 11724, USA and \\ 2 Molecular and Cellular Biology Program, Stony Brook University, Stony Brook, \\ NY 11794, USA \\ * Corresponding author: Y Lazebnik, Cold Spring Harbor Laboratory, One \\ Bungtown Rd, Cold Spring Harbor, NY 11724, USA. Tel: 516367 8357; \\ Fax: 516367 8461; E-mail: lazebnik@cshl.edu
}

Received 02.11.05; revised 28.3.06; accepted 10.4.06; published online 26.5.06 Edited by GM Cohen

\section{Abstract \\ During apoptosis, cytochrome $c$ released from mitochondria activates Apaf-1, a cofactor of caspase-9. The evidence that cytochrome $c$ can activate Apaf- 1 is abundant, but the proof that cytochrome $c$ is required for apoptosis is limited to two studies that used genetically modified mice. One of these studies concluded that in some tissues apoptosis may require Apaf-1 but not cytochrome $c$, which indicated the need to analyze the requirement of cytochrome $c$ beyond the mouse models, and in human tumor cells in particular. In this study, we designed tools to silence cytochrome $c$ expression in human cells and tested these tools in an experimental system of oncogenic transformation. We found that cytochrome $c$ was required for apoptosis induced by both DNA damage and, unexpectedly, TNF $\alpha$. Overall, this study established that cytochrome $c$ is required for apoptosis in human cells and provided tools to dissect mechanisms of apoptosis in various experimental models. \\ Cell Death and Differentiation (2006) 13, 2062-2067. \\ doi:10.1038/sj.cdd.4401968; published online 26 May 2006}

Keywords: apoptosis; cytochrome $c$; cancer; DNA damage

Abbreviations: apaf-1, apoptotic protease-activating factor-1; Bax, Bcl-2-associated X protein; Bcl-2, B-cell lymphoma 2; Bid, $\mathrm{Bcl}-2$ interacting domain death agonist; $\mathrm{CHX}$, cycloheximide; CrmA, cytokine response modifier A; Dark, Drosophila Apaf1-related killer; EGFP, Enhanced green fluorescence protein; FlipL, FLICE inhibitory protein long; RNAi, RNA interference; siRNA, small interfering RNA; Smac, Second mitochondria derived activator of caspase; TNF, tumor necrosis factor; TRAIL, tumor necrosis factor-related apoptosis-inducing ligand

\section{Introduction}

Many cancer chemotherapeutics induce apoptosis and many are being developed to do so. ${ }^{1}$ Therefore, knowing how apoptotic pathways in cancer cells are organized may help to improve treatment efficiency, or to avoid unnecessary treatment or drug development. Although the general design of the apoptotic machinery is conserved throughout the species and tissues, the possibility of undiscovered mechanisms ${ }^{2,3}$ the inherently high heterogeneity of cancers, ${ }^{4}$ and the very abnormality of cancer cells imply that apoptotic pathways of these cells may vary substantially. This variability may mean that apoptotic pathways of each tumor or its subpopulation may need to be mapped to optimize treatment and to learn how diverse organization of these pathways is. Approaches that exploit RNAi are especially attractive for such mapping because they are rapid and work in human cells.

During apoptosis, cells are disassembled by caspases, a family of proteases that are activated by two converging pathways. In the extrinsic pathway, binding of cytokines to their receptors results in activation of caspase-8, which then activates caspase-3, the protease that cleaves the majority of caspase substrates thereby disassembling the cell. ${ }^{5}$ In the intrinsic pathway, a variety of stimuli, including DNA damage, regulate the $\mathrm{Bcl}-2$ family of proteins that control release of cytochrome $c$ and other proteins from mitochondria. Once released, cytochrome $c$ binds and activates Apaf-1, a cofactor of caspase-9, a protease that then processes and activates caspase- $3 .{ }^{6}$ In the so-called Type II cells, ${ }^{7}$ apoptosis induced by activation of TRAIL receptor or Fas is initiated by the extrinsic pathway but is completed by the distal branch of the intrinsic pathway. In these cells, activated caspase-8 for some reason poorly processes caspase-3, but efficiently cleaves a $\mathrm{Bcl}-2$ family protein Bid. One of the resulting Bid fragments releases cytochrome $c$ from mitochondria thereby activating caspase- 9 and, consequently, caspase- 3 .

The ability of cytochrome $c$ to activate Apaf-1 is supported by numerous observations, ${ }^{6}$ although the requirement of cytochrome $c$ for apoptosis in mammalian cells has been tested only in two studies. The first study ${ }^{8}$ found that the deficiency of cytochrome $c$ in embryonic cells inhibited apoptosis induced through the intrinsic but not the extrinsic pathway, and increased clonogenic survival of the cells following drug treatment. Perhaps because cytochrome $c$ is required for mitochondrial respiration, mice deficient in this protein die early in development, restricting the quantity and variety of cells available for analysis.

To overcome this problem, a more recent study ${ }^{9}$ used mice in which cytochrome $c$ was substituted with a mutant (cytochrome KA) that functions in respiration but has reduced ability to activate Apaf-1. ${ }^{10,11}$ This study confirmed the requirement of cytochrome $c$ for the intrinsic pathway of apoptosis, but found that the phenotypes of the cytochrome $\mathrm{KA}$ and mice deficient in Apaf-1 overlapped only partially, which led to the conclusion that in some tissues cytochrome $c$ may not be required for Apaf-1-dependent apoptosis. If true, this conclusion would imply undiscovered pathways of Apaf-1 activation, perhaps analogous to those that activate Drosophila Apaf-1 orthologue Dark, which may function without cytochrome $c .^{12}$ In particular, this possibility raises the need to compare the requirements for Apaf-1 and cytochrome $c$ beyond the mouse models. 
In this study, we developed tools to test the requirement for cytochrome $c$ for apoptosis in human cells and compared the effects of cytochrome $c$ and Apaf- 1 deficiencies on apoptosis and cell survival.

\section{Results and Discussion}

To test whether cytochrome $c$ is required for apoptosis in human cells, we used human lung fibroblasts that express adenoviral oncogene $\mathrm{E} 1 \mathrm{~A}\left(\mathrm{IMR} 90^{\mathrm{E} 1 \mathrm{~A}}\right)$, a cell line that has been used as a convenient and informative model to study effects of oncogenes on mechanisms of apoptosis. In these cells, silencing Apaf-1 or expression of a caspase-9 dominantnegative mutant prevents caspase- 9 activation and the subsequent cell disassembly. ${ }^{13,14}$ Therefore, if cytochrome $c$ is required for activating Apaf-1 and apoptosis, then silencing expression of either protein should have the same effect.

Because the reported half-life of cytochrome $c$ in cells is 8 days, ${ }^{15}$ we first tested whether concentration of this protein can be efficiently decreased by RNAi. We found that cytochrome $c$ was barely detectable by immunoblotting in 4 days after transfecting cells with either of the two siRNAs that we designed (siCc1 and siCc2, Materials and Methods) (Figure 1a). The efficiency of silencing varied among experiments, with siCc2 being always less effective than siCc1. The silencing was not owing to the transfection procedure itself as an siRNA that we used to control for general effects of siRNA transfection (siC1), or an siRNA against Apaf-1 (siA1) ${ }^{13}$ had no effect on cytochrome $c$ expression (Figure 1a).

Cytochrome $c$ remained detectable by immunofluorescence in cells transfected with siCc2, and, to a lesser degree, with siCc1 (Figure 1b), indicating again that siCc2 was less effective than siCc1. According to this assay, cytochrome $c$ was silenced by siCc1 in about $70 \%$ of cells (unpublished results). The majority of cells transfected with either siCc1 or siCc2 remained viable, but transfection with siCc1 caused death of about $10 \%$ of the cells shortly after transfection. Transfected cells grew in tissue culture medium for several days with no additional supplements (Materials and Methods), but the rate of proliferation decreased following transfection of siCc1 (unpublished results).

\section{Cytochrome $c$ is required for apoptosis induced by DNA damage}

To determine whether cytochrome $c$ was required for apoptosis, we transfected IMR90 ${ }^{\mathrm{E} 1 \mathrm{~A}}$ cells with siA1, siC1, siCc1, or siCc2, treated the cells with etoposide, an anticancer a
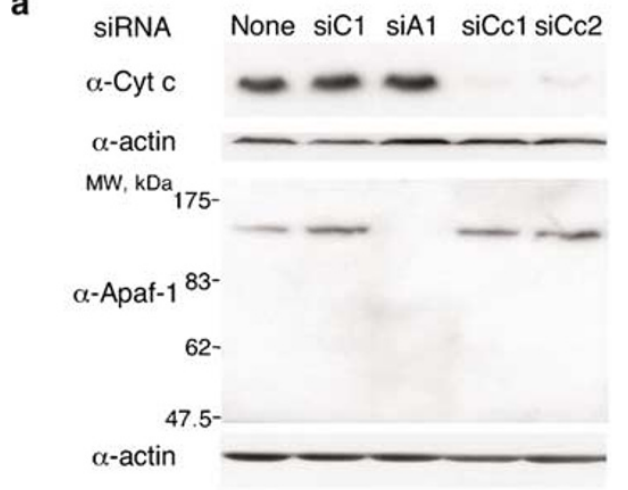

b

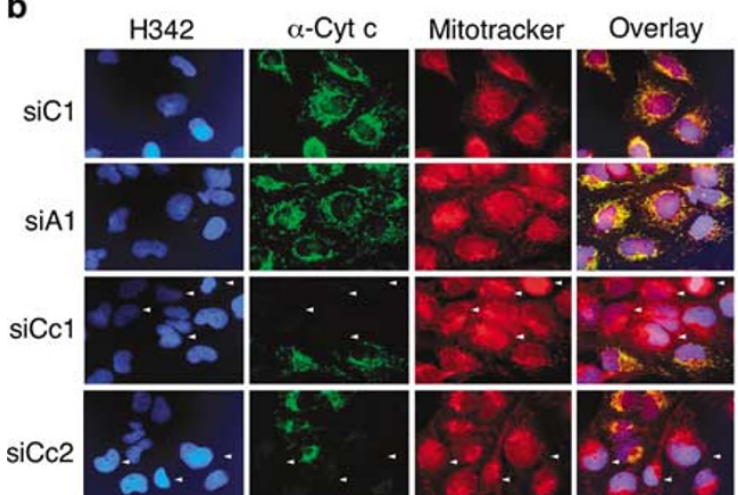

C

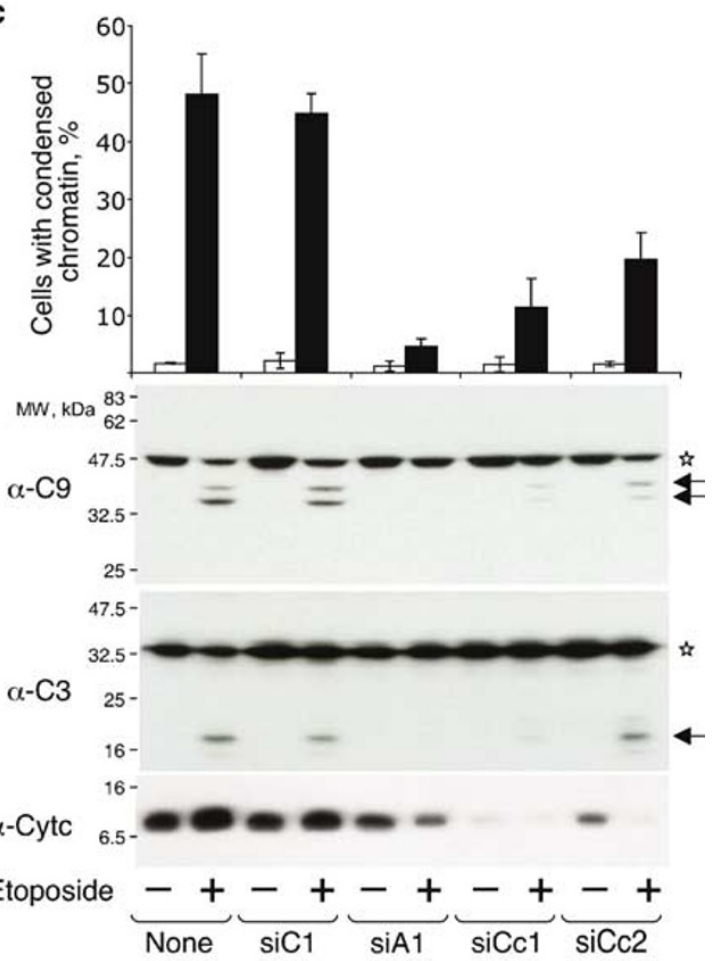

Figure 1 Cytochrome $c$ and Apaf-1 are required for apoptosis induced by DNA damage. (a) IMR90 ${ }^{\mathrm{E} A \mathrm{~A}}$ cells were transfected with siRNAs to caspase-1 (siC1), Apaf-1 (siA1), cytochrome $c$ (siCc 1 and siCc2), or left untransfected (None). Four days after transfection, expression of cytochrome $c$ and Apaf-1 was analyzed by immunoblotting (Materials and Methods). An equal amount of total protein was loaded in each lane, and loading was verified by re-probing blots with an antibody to $\beta$-actin. (b) Cells transfected with indicated siRNAs as in (a) were stained with an antibody to cytochrome $c$ and mitochondria were visualized with Mitotracker Red and DNA with Hoechst 33342. Arrowheads indicate cells with decreased cytochrome $c$ expression. (c) IMR90 ${ }^{\mathrm{E} 1 \mathrm{~A}}$ cells transfected with indicated siRNAs were treated with $50 \mu \mathrm{M}$ etoposide (solid bars) or left untreated (open bars) for $22 \mathrm{~h}$, collected and used to score apoptotic cells using chromatin condensation as a marker, and to visualize processing of caspase-9 and caspase-3 by immunoblotting (Materials and Methods). Asterisks indicate the precursors whereas arrows indicate the processed caspases. The quantification data represent averages of three independent experiments with the error bars indicating standard deviations. The immunoblots are from one of the experiments 
drug that induces DNA breaks, and monitored apoptosis by observing chromatin condensation. siA1, siCc1, or siCc2 inhibited apoptosis, whereas siC1 had little effect (Figure 1c). Similar effects were observed in HeLa cells (Supplementary Figure 1), implying that the effects of silencing were not restricted to $\mathrm{IMR} 90^{\mathrm{E} 1 \mathrm{~A}}$ cells. siA1 and $\mathrm{siCc} 1$ inhibited processing of caspase- 9 and its substrate caspase-3 in IMR90 ${ }^{\mathrm{E} 1 \mathrm{~A}}$ cells (Figure 1c), confirming that Apaf-1 and cytochrome $c$ are both required for caspase-9 activation. Consistent with the difference in the effectiveness of silencing, siCc1 inhibited apoptosis and caspase-9 processing better than siCc2, which may explain why some caspase-3 was still processed in cells transfected with siCc2 (Figure 1c).

The similarity between the effects of siCc1 and siCc2, and the direct correlation between their effectiveness in silencing and the ability to inhibit apoptosis was consistent with the conclusion that the effect of these siRNAs on apoptosis is due to silencing cytochrome $c$. To verify this conclusion, we attempted to rescue the effect of these siRNAs by expressing cytochrome $c$ ectopically. However, cytochrome $c$ constituted only a minor fraction of the endogenous protein in all numerous cell lines that we generated. The intriguing difficulty with expressing cytochrome $c$ was consistent with previous report ${ }^{16}$ but remains unexplained. We found that inhibiting the proteasome increased the amounts of the ectopic cytochrome $c$ to the desirable levels, but the protein failed to enter mitochondria and aggregated in the nucleus (unpublished results). Eventually, we used cell lines that expressed a fusion of cytochrome $c$ and EGFP, but found that the rescue effect was marginal, perhaps because the achieved concentration of the fusion protein was insufficient to induce apoptosis (unpublished results).

The observation that cytochrome $c$ concentration had to be decreased many fold to inhibit apoptosis raised the possibility that substitution of cytochrome $c$ with the KA mutant, ${ }^{9}$ whose ability to activate Apaf- 1 is reduced 12 -fold, ${ }^{10}$ may not be equivalent to deleting the apoptotic function of cytochrome $c$. Perhaps using mutants that are unable to activate Apaf- 1 at all ${ }^{10}$ would determine whether this possibility is true.

\section{Cytochrome $c$ is required for TNF $\alpha$-induced apoptosis}

As an approach to test the specificity of silencing cytochrome $c$, we used the observation that mouse embryonic cells deficient in cytochrome $c$ remain sensitive to TNF $\alpha{ }^{8}$ This observation is consistent with the requirement of cytochrome $c$ for the intrinsic but not the extrinsic pathway of apoptosis, and predicted that silencing cytochrome $c$ should not affect apoptosis induced by TNF $\alpha$ in our experimental system. Indeed, siCc2 had no statistically significant effect on apoptosis (Figure 2a), although cytochrome $c$ silencing with this siRNA was particularly ineffective in these experiments (Figure 2b). However, siCc1, which silenced more effectively, inhibited apoptosis (Figure 2a), implying that either this siRNA had an unidentified target involved in the extrinsic pathway, or caspase- 9 activation was required for $\operatorname{TNF} \alpha$ induced apoptosis.

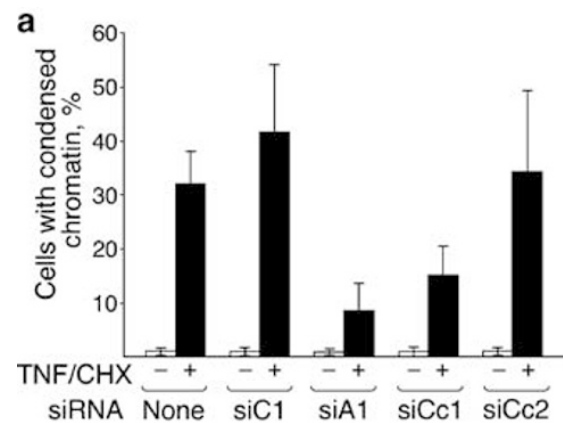

d

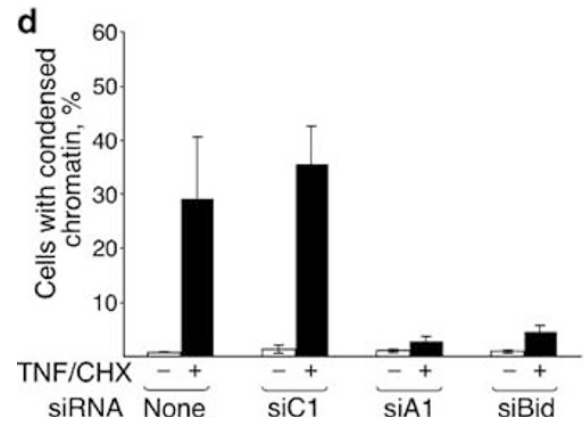

b

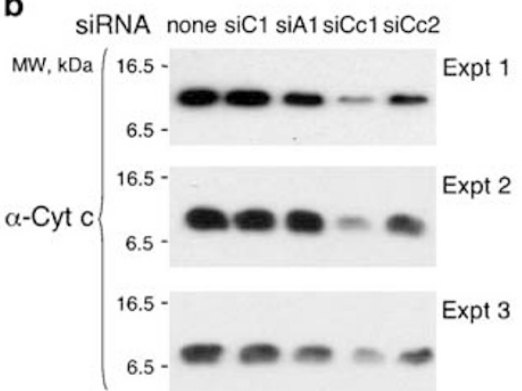

e

siRNA none siC1 siA1 siBid

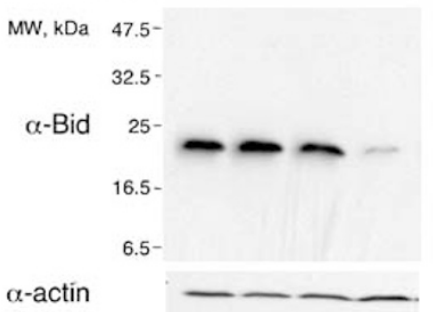

c

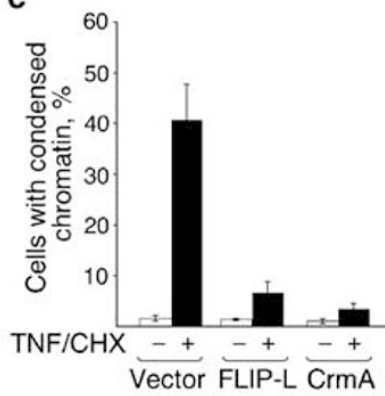

f

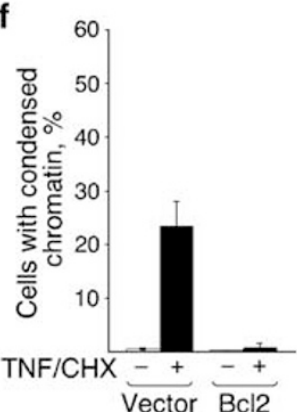

Figure 2 Cytochrome $c$ is required for apoptosis induced by TNF $\alpha$. (a) IMR $90^{\mathrm{E} 1 \mathrm{~A}}$ cells transfected with indicated siRNAs were treated with $35 \mathrm{ng} / \mathrm{ml} \mathrm{TNF} \alpha$ and $6 \mu \mathrm{g} / \mathrm{ml}$ cycloheximide (solid bars) or left untreated (open bars) for $24 \mathrm{~h}$, and collected to score apoptosis by counting cells with condensed chromatin. Expression of cytochrome $c$ in these experiments was evaluated by immunoblotting (b). (c) IMR90 ${ }^{\mathrm{E} 1 \mathrm{~A}}$ cell lines that expressed indicated proteins were treated and processed as in (a). (d) IMR9 ${ }^{\mathrm{E} 1 \mathrm{~A}}$ cells were transfected with indicated siRNAs and processed as in (a), and the effect of siRNA to Bid (siBid) was monitored by immunoblotting (e). (f) IMR90 ${ }^{\mathrm{E} 1 \mathrm{~A}}$ or the cells that expressed Bcl-2 were treated and processed as in (a). The numerical data (in $\mathbf{a}, \mathbf{c}, \mathbf{d}$, and $\mathbf{f}$ ) are averages of three independent experiments with the error bars indicating standard deviations 
We reasoned that if the second possibility was true, then the sequence of events leading to cell death should be similar to that observed in type II cells. In these cells, TRAIL or agonists of Fas activate caspase-8, which for some reason fails to activate caspase- 3 but processes Bid, which releases cytochrome $c$ leading to activation of caspase-9, and, consequently, of caspase-3. Therefore, we tested whether this sequence of events mediated TNF $\alpha$-induced apoptosis in IMR90 ${ }^{\mathrm{E} 1 \mathrm{~A}}$ cells.

Consistent with the requirement for caspase- 8 in TNF $\alpha$ induced apoptosis, ectopic expression of FlipL, an inhibitor of caspase-8 activation, or of crmA, an inhibitor of caspase-8 activity, protected the cells from TNF $\alpha$ (Figure 2c), and prevented caspase-8 and caspase-9 processing (Supplementary Figure S2A). Apoptosis was also inhibited by silencing Bid (Figure $2 \mathrm{~d}$ and e), indicating that Bid is required, and by ectopically expressed $\mathrm{Bcl}-2$, which protects mitochondria from the effect of Bid (Figure 2f). Finally, apoptosis was inhibited by silencing Apaf-1 (Figure 2d). Altogether, these observations indicated that apoptosis induced by TNF $\alpha$ proceeds through the sequence of events described for type II cells. Therefore, the inhibitory effect of siCc1 on this apoptosis induced by TNF $\alpha$ could be explained by silencing of cytochrome $c$.

\section{Silencing cytochrome $c$ but not Apaf-1 increases clonogenic survival following DNA damage}

Mouse cells deficient in Apaf-1 or caspase- 9 are not disassembled following activation of the intrinsic pathway, but they do not proliferate, ${ }^{17}$ whereas cells deficient in cytochrome $c$ do. ${ }^{8}$ In principle, this difference may mean that the role of cytochrome $c$ in cell survival is not identical to that of Apaf-1 or caspase-9, or that cells deficient in these three proteins are also different in some other respects, which is not a remote possibility considering that the cells are derived from different knockout mice.

We took advantage of the opportunity provided by using siRNA to compare effects of either cytochrome $c$ or Apaf-1 deficiency in the same cell line. We found that although silencing expression of either protein decreased the rate of cell death induced by DNA damage (Figure 3a), only a deficiency in cytochrome $c$ resulted in clonogenic survival of the treated cells (Figure $3 b$ ). This difference implied that cytochrome $c$ deficiency delayed caspase- 9 activation better than a deficiency in Apaf-1, which was contrary to our observations (Figure 1c), that the lack of cytochrome $c$ affected apoptosis at a stage that does not require Apaf-1, or that the effects of siCc1 are not restricted to silencing the target gene.

To test whether siCc1 affects apoptosis in a way different from activating Apaf-1, we tested whether this siRNA affected the pathways that lead to the release of proteins from mitochondria. To monitor this release, we visualized the release of Smac, a mitochondrial protein involved in apoptosis, by immunofluorescence. To facilitate the assay, we used IMR90 ${ }^{\mathrm{E} 1 \mathrm{~A}}$ cells in which caspase-9 activation and the consequent cell disassembly were prevented by expressing caspase- 9 dominant-negative mutant (C9DN). ${ }^{14}$
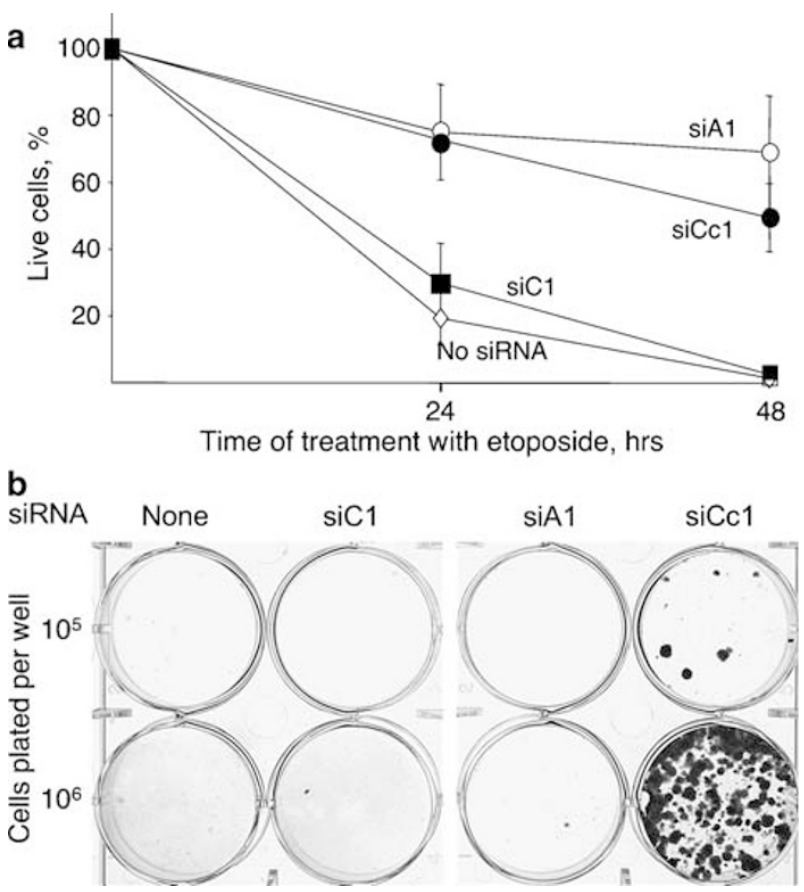

Figure 3 Silencing cytochrome $c$ but not Apaf- 1 increases clonogenic survival of cells subjected to DNA damage. (a) Cells were transfected with indicated siRNAs for 4 days and then treated with $50 \mu \mathrm{M}$ etoposide for indicated time, and scored for viability. Cells that remained adherent and also excluded Trypan blue were counted as live. To compare effects of the siRNAs, the initial number of live cells in each experiment was assigned to be $100 \%$. The data are averages of three independent experiments with the error bars indicating standard deviations. (b) Cells transfected as in (a) were treated with etoposide for $24 \mathrm{~h}$, then cells that remained adherent were collected and plated at indicated density using medium without etoposide (Materials and Methods). After 14 days, the adherent cells were fixed and stained with Crystal Violet to visualize cell colonies. The presented plates are from one of three experiments all of which gave similar results

siCc1 decreased the number of cells that released Smac (Figure $4 \mathrm{a}$, top panel, and $4 \mathrm{~b}$ ), whereas an siRNA to Smac had no effect on release of cytochrome $c$ (Figure $4 a$, middle panel, and Supplementary Figure S2B). Therefore, siCc1 inhibited mitochondrial permeabilization, perhaps by changing the sensitivity of cells to DNA damage. This explanation was also consistent with the inhibitory effect on translocation of Bax to mitochondria (Figure 4a, bottom panel, and $4 b$ ).

siCc 1 could change the sensitivity to DNA damage either by causing a deficiency in cytochrome $c$ or by an unidentified offtarget effect. For example, a deficiency of cytochrome $c$ may cause a deficiency in mitochondrial respiration, which, in turn, could have an effect on the rate of proliferation. Indeed, we noticed that cells transfected with siCc1 grew somewhat slower than cells transfected with other siRNAs (unpublished results). However, we found no differences between cell cycle distribution of untransfected cells, and cells transfected with either siC1 or siCc1 (Supplementary Figure S3). Interestingly, siA1 increased the fraction of cells on S-phase of cell cycle, although whether this effect is owing to silencing of Apaf-1 is unclear. Following DNA damage, cell cycle distributions of cells transfected with siA1 or siCc1 were also similar. We also found no effect of siCc1 on induction of expression of p21 or phosphorylation of p53 by DNA damage (unpublished results). 

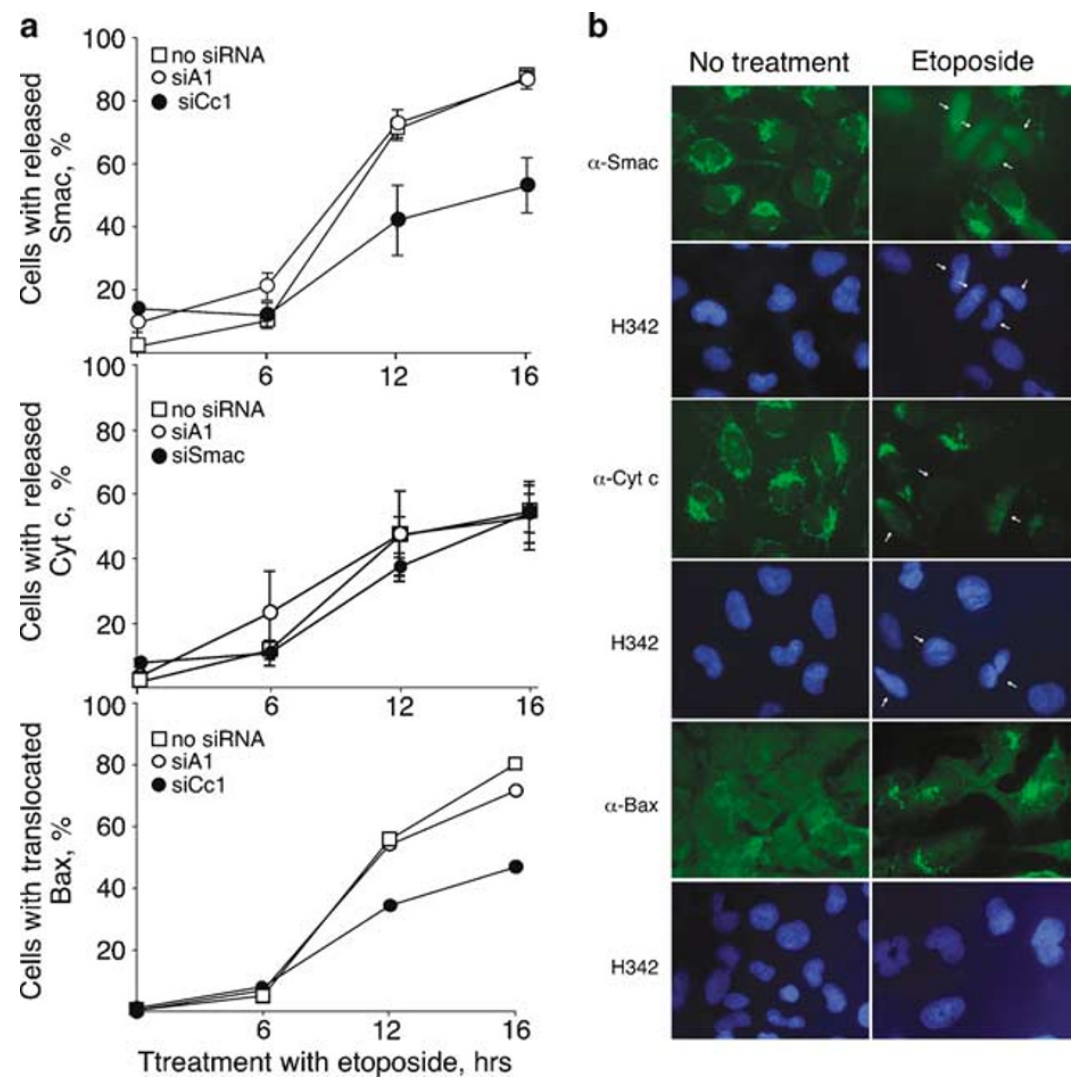

Figure 4 Silencing cytochrome $c$ affects sensitivity to DNA damage. (a) IMR90 ${ }^{E 1 A}$ cells that expressed C9DN were transfected with indicated siRNAs or left untransfected, and then treated with etoposide for indicated time. The cells were scored for release of Smac (top panel), cytochrome $c$ (middle panel), or translocation of Bax to the mitochondria (bottom) by immunofluorescence, as indicated in (b). The data in (a) represent average of three experiments with the error bars indicating standard deviations, except the data on Bax translocation (a, bottom panel), which are an average of two experiments. The arrows in (b) indicate cells with released Smac or cytochrome $c$

Overall, our observations failed to reveal any effect of siCc1 on DNA damage response but did not rule out this effect. Testing this possibility would require expression of sufficient amounts of ectopic cytochrome $c$, or another siRNA that is as effective in silencing cytochrome $c$ as is siCc1. Unfortunately, we were unsuccessful in implementing either approach. A formal possibility, which also remains to be tested, is that cytochrome $c$ is required for mitochondrial permeabilization.

Overall, we found that a deficiency in cytochrome $c$ is required for activation of caspase- 9 and apoptosis in human cells, that in some cells apoptosis induced by TNF $\alpha$ requires activation of caspase- 9 , and that silencing expression of cytochrome $c$ increased long-term cell survival. We hope that the tools developed during this study will be useful for evaluating the diversity of apoptotic pathways in human tumor cells.

\section{Materials and Methods}

\section{Tissue culture}

All cells were maintained in Dulbecco's modified minimal medium (Invitrogen Cat \# 11995-065) supplemented with 10\% FBS.

\section{siRNA design and transfection}

All siRNAs were purchased from Dharmacon. GUACAUCCCUGGAAC AAAA (siCc1) was targeted to the coding region of cytochrome $c$ and
UUGGCC ACUGCCUUAUUUA (siCc2) to $3^{\prime}$ UTR of the cytochrome $c$ mRNA. siRNA to human Bid (siBid) was AGACAUCAUCCGGAAUAUU and siRNA to human Smac (siSmac) was GAAGCGGUGUUUCUCAGAA. siRNAs to Apaf- 1 and caspase- 1 have been described previously. ${ }^{13}$ Cells were transfected with $100 \mathrm{nM}$ siRNA unless otherwise indicated using Optimem according to the manufacturer's instructions using the protocol described previously. ${ }^{18}$

\section{Gene transduction}

Bcl2, CrmA, and Flip-L were cloned into the pMaRXIVpuro ${ }^{19}$ vector. All cell lines were made by retroviral transductions as previously described. ${ }^{14}$ Briefly, plasmids were transiently transfected into packaging cell line to produce retrovirus, which was used to infect IMR $90^{\mathrm{E} 1 \mathrm{~A}}$ cells. The infected cells were selected using a corresponding drug. The infection efficiency was typically about $80-90 \%$. After the study was completed, we found that the Bcl-2 cDNA had a mutation that converted glycine 237 into serine. Whether this mutation affects $\mathrm{Bcl}-2$ function is unclear, but it did not eliminate its inhibitory effect on apoptosis.

\section{Antibodies}

The monoclonal antibodies to caspase-9, caspase-8, Apaf-1, and Smac have been described previously. ${ }^{13,14}$ Monoclonal antibodies to actin (Santa Cruz), cytochrome $c$ and Bax (BD Pharmingen), and polyclonal antibody for human Bid (Cell Signaling) were purchased from the indicated suppliers. 


\section{Detection of cytochrome $c$ and Smac by immunofluorescence}

Cytochrome c, Smac, and Bax were detected with the monoclonal antibodies as described perviously ${ }^{13}$ and visualized with anti-mouse Alexa 488. To visualize the mitochondria, cells were incubated at $37^{\circ} \mathrm{C}$ with Mitotracker Red (Molecular Probes) 30 min before fixation according to the manufacturer's instructions.

\section{Scoring apoptotic cells}

All cells, already detached and still adherent, were collected, combined, fixed in $4 \%$ paraformaldehyde, stained with $1 \mu \mathrm{g} / \mathrm{ml}$ Hoechst 33342, and cells with condensed chromatin were scored as apoptotic using a fluorescence microscope. A total of $200-500$ cells were counted for each sample.

\section{Clonogenic survival}

Cells were transfected with siC1, siApaf-1, or siCc1 and treated in 4 days with $50 \mu \mathrm{M}$ etoposide for $24 \mathrm{~h}$. Cells that remained adherent were rinsed with PBS, collected by trypsinization and replated in fresh medium without etoposide at a density of $10^{5}$ or $10^{6}$ cells per well of a six-well plate. After 14 days, the attached cells were washed with PBS, fixed, and stained with Crystal Violet to visualize cell colonies.

\section{Cell cycle analysis}

Cells were transfected with siC1, siApaf-1 or siCc1, or left untransfected and treated after 4 days with $50 \mu \mathrm{M}$ etoposide for $16 \mathrm{~h}$. Untreated cells and treated cells were stained with DAPI $(10 \mu \mathrm{g} / \mathrm{ml})$ for $15 \mathrm{~min}$ on ice and analyzed for DNA content using Becton Dickinson LSR-II Cell Analyzer. Cell cycle distribution was calculated using Modfit LT software (Verity).

\section{Acknowledgements}

We thank Joe Rodriguez for designing siCc1 and providing the expression plasmid with FLIP-L, Patrice Lassus for designing siSmac and providing CDNA for Bcl-2 and crmA, Jack Lin for providing IMR90 ${ }^{\mathrm{E} 1 \mathrm{~A}}$ cells, and Dominik Duelli for providing the corresponding cells that express caspase9 dominant-negative mutant. We thank Ximena Opitz-Araya for help with cloning, and Cathy Cormier, Dom Duelli, and Jack Lin for comments on the manuscript. This study was supported by the NIH Grant CA-13106-31 and by the Maxfield Foundation.

\section{References}

1. Kaufmann SH and Vaux DL (2003) Alterations in the apoptotic machinery and their potential role in anticancer drug resistance. Oncogene 22: 7414-7430.
2. Marsden VS, Ekert PG, Van Delft M, Vaux DL, Adams JM and Strasser A (2004) Bcl-2-regulated apoptosis and cytochrome $c$ release can occur independently of both caspase-2 and caspase-9. J. Cell Biol. 165: 775-780.

3. Adams JM (2003) Ways of dying: multiple pathways to apoptosis. Genes Dev. 17: 2481-2495.

4. Bignold LP (2004) Chaotic genomes make chaotic cells: the mutator phenotype theory of carcinogenesis applied to clinicopathological relationships of solid tumors. Cancer Invest. 22: 338-343.

5. Riedl SJ and Shi Y (2004) Molecular mechanisms of caspase regulation during apoptosis. Nat. Rev. Mol. Cell Biol. 5: 897-907.

6. Jiang $X$ and Wang $X$ (2004) Cytochrome $C$-mediated apoptosis. Annu. Rev. Biochem. 73: 87-106.

7. Scaffidi C, Fulda S, Srinivasan A, Friesen C, Li F, Tomaselli KJ, Debatin KM, Krammer PH and Peter ME (1998) Two CD95 (APO-1/Fas) signaling pathways. EMBO J. 17: 1675-1687.

8. Li K, Li Y, Shelton JM, Richardson JA, Spencer E, Chen ZJ, Wang X and Williams RS (2000) Cytochrome $c$ deficiency causes embryonic lethality and attenuates stress-induced apoptosis. Cell 101: 389-399.

9. Hao Z, Duncan GS, Chang CC, Elia A, Fang M, Wakeham A, Okada H, Calzascia T, Jang Y, You-Ten A, Yeh WC, Ohashi P, Wang X and Mak TW (2005) Specific ablation of the apoptotic functions of cytochrome $c$ reveals a differential requirement for cytochrome $C$ and Apaf-1 in apoptosis. Cell 121: 579-591.

10. Abdullaev Z, Bodrova ME, Chernyak BV, Dolgikh DA, Kluck RM, Pereverzev MO, Arseniev AS, Efremov RG, Kirpichnikov MP, Mokhova EN, Newmeyer DD, Roder $\mathrm{H}$ and Skulachev VP (2002) A cytochrome $c$ mutant with high electron transfer and antioxidant activities but devoid of apoptogenic effect. Biochem. J. 362: 749-754.

11. Kluck RM, Ellerby LM, Ellerby HM, Naiem S, Yaffe MP, Margoliash E, Bredesen D, Mauk AG, Sherman F and Newmeyer DD (2000) Determinants of cytochrome $c$ pro-apoptotic activity. The role of lysine 72 trimethylation. J. Biol. Chem. 275: 16127-16133.

12. Kornbluth $\mathrm{S}$ and White $\mathrm{K}$ (2005) Apoptosis in Drosophila: neither fish nor fowl (nor man, nor worm). J. Cell. Sci. 118: 1779-1787.

13. Lassus $P$, Opitz-Araya $X$ and Lazebnik $Y$ (2002) Requirement for caspase-2 in stress-induced apoptosis before mitochondrial permeabilization. Science 297: 1352-1354.

14. Fearnhead HO, Rodriguez J, Govek EE, Guo W, Kobayashi R, Hannon G and Lazebnik YA (1998) Oncogene-dependent apoptosis is mediated by caspase9. Proc. Natl. Acad. Sci. USA 95: 13664-13669.

15. Terjung RL (1979) The turnover of cytochrome $c$ in different skeletal-muscle fibre types of the rat. Biochem J. 178: 569-574.

16. Goldstein JC, Waterhouse NJ, Juin P, Evan GI and Green DR (2000) The coordinate release of cytochrome $c$ during apoptosis is rapid, complete and kinetically invariant. Nat. Cell Biol. 2: 156-162.

17. Ekert PG, Read SH, Silke J, Marsden VS, Kaufmann H, Hawkins CJ, Gerl R, Kumar S and Vaux DL (2004) Apaf-1 and caspase-9 accelerate apoptosis, but do not determine whether factor-deprived or drug-treated cells die. J. Cell Biol. 165: 835-842.

18. Lassus $P$, Rodriguez $J$ and Lazebnik $Y$ (2002) Confirming specificity of RNAi in mammalian cells. Sci. STKE 2002: PL13.

19. Hannon GJ, Sun P, Carnero A, Xie LY, Maestro R, Conklin DS and Beach D (1999) MaRX: an approach to genetics in mammalian cells. Science 283: $1129-1130$.

Supplementary Information accompanies the paper on Cell Death and Differentiation website (http://www.nature.com/cdd) 